\title{
Lohngestaltung in der Tschechischen Republik
}

\author{
Zuzana Dvoráková*
}

Die Lohngestaltung in der Tschechischen Republik wurde in der ersten Hälfte der 90er Jahre von der Entwicklung des Arbeitsmarktes nicht beeinflußt. Bis 1997 war für die Wirtschaft ein langjähriger Trend des schnelleren Wachstums der Nominallöhne im Vergleich zu der Produktivitätssteigerung charakteristisch wie die nicht vollendete Restrukturalisierung der Industrie und die Privatisierung der Banken. Umfangreiche Umverteilungen der Arbeitskräfte verliefen bei einer sehr niedrigen Arbeitslosigkeit. Deshalb wurden sie durch den Druck auf die Kostensenkung sowie Produktivitätssteigerung nicht begleitet.

Die Lohnsysteme wurden durch die Entwicklung der Legislative im Bereich der arbeitsrechtlichen Beziehungen, der Vergütung sowie der Tarifverhandlungen determiniert. Die Novellierung des Arbeitsgesetzbuches und das schnelle Erlassen des Gesetzes Nr. 1/1992 Slg., über den Lohn, die Vergütung für den Bereitschaftsdienst und den durchschnittlichen Lohn bildeten günstige Voraussetzungen dafür, daß die Unternehmen neue Lohnsysteme bilden und entwickeln konnten, die die Prinzipien der Marktwirtschaft respektieren. Den rechtlichen Rahmen bildete das Gesetz Nr. 2/1991 Slg., über die Tarifverhandlung. Der Lohn wurde auf dem Vertragsprinzip festgesetzt und bot den Arbeitgebern eine Autonomie in der Lohnpolitik und bei der Gestaltung der Lohnsysteme. Dies ermöglichte, die langjährigen Folgen des zentral geleiteten Systems der Vergütung zu überwinden, in dem das entscheidende Instrument das Tarifsystem darstellte. Die einzige Einschränkung stellen die in den Tarifverträgen vereinbarten Barrieren dar. Nach den Erfahrungen mit dem zentralen Vergütungssystem waren die Unternehmen generell bemüht, transparente Lohnsysteme anzuwenden.

Bei der Änderung des Lohngefälles, das sich im Laufe der Planwirtschaft herausbildete, spielten ausländische Gesellschaften, neu privatisierte Unternehmen und Banken eine entscheidende Rolle (Flanagan 1995; Klain 1995). Sie nützten die legislativen Normen aus und starteten den Prozeß einer positiven Veränderung der Lohngestaltung in der Volkswirtschaft (Mzdy 1997; Mzdy 1998). Die einheimischen Arbeitgeber waren nicht in der Lage, das

\footnotetext{
* Dvorákova, Zuzana, (* 1960), Wirtschaftsuniversität Prag, Lehrstuhl für Personalmanagement. Forschungs-/ Arbeitsschwerpunkte: Tarifverhandlungen und Vergütung.
} 
Zurückbleiben ihres Lohnniveaus hinter den Unternehmen mit dem Auslandskapital wettzumachen. Die Regulierung der Lohn- und Gehaltssumme verlangsamte eine Normalisierung des Lohngefälles im Sinne der Entlohnung nach dem Arbeitswert und nach der Leistung des Arbeitnehmers. Die Regulierung wirkte sich als eine wirksame Maßnahme gegen die Inflation aus, aber sie übte einen negativen Einfluß auf die Unternehmen aus, weil sie den Zuwachs des durchschnittlichen Lohnes durch die Entwicklung der Verbraucherpreise einschränkte. Auf diese Weise wurde sie zu einem Hindernis für die Rationalisierung der Beschäftigung in den Unternehmen, für die Freistellung von weniger produktiven Arbeitnehmern und die Lohngestaltung anhand der Produktivität. Insgesamt verlangsamte sie den Prozeß der Verschiebung der Arbeitskräfte in Richtung zu den produktiveren Unternehmen und Branchen. Ihre Auflösung im Jahre 1995 wurde von den Unternehmern begrüßt.

Die Erfahrungen der Funktion arbeitsrechtlicher Normen bringen gegenwärtig das Anliegen mit sich, deren Einfluß auf die Einstellung zur Vergütung der jeweiligen Unternehmen $\mathrm{zu}$ beurteilen, damit die Vorschläge zu ihrer Novellierung bearbeitet werden können. Im Gegensatz zu den Richtlinien der Europäischen Union ist in unserem Lohngesetz das Prinzip des gleichen Lohnes für die Arbeit des gleichen Wertes nicht ausdrücklich verankert, obwohl die Beseitigung der Lohndiskriminierung von Frauen und Minderheiten ein Anreiz zum Produktivitätswachstum sein kann.

Der vorliegende Beitrag setzt sich zum Ziel, die Lohngestaltung und-systeme in der Tschechischen Republik in den Jahren 1990-1998 zu beurteilen. Dies könnte eine wertvolle Diskussion anregen, denn das Thema der Entlohnung in einer sich transformierenden Wirtschaft wird in den publizierten Artikeln nicht häufig behandelt.

Die Bewertung der Lohnpolitik knüpft an die Ergebnisse der nationalen Untersuchung über das Human Resource Management (HRM) in der Tschechischen Republik in den Jahren 1996 und 1998 an. Diese Untersuchung wurde vom Lehrstuhl für das Personalmanagement der Wirtschaftsuniversität in Prag im Rahmen des Cranfield-Projektes über das Europäische Management von Humanressourcen durchgeführt. Das Projekt analysierte die Transformation des HRM. Die Umfrage wurde nach der Methodik des Centre for European HRM der Cranfielder Universität in Großbritannien vorbereitet. Der Fragebogen beinhaltete 6 Teile und wurde durch einen siebten Teil ergänzt, der sich ausführlich mit den Lohnsystemen befaßte. Im Jahre 1996 wurde die Umfrage an 2000 Organisationen versandt, die zufällig dem Register der Informationseinheiten des Tschechischen Statistischen Amtes entnommen wurden. Ihre Zusammensetzung kopierte die Branchenstruktur der Volkswirtschaft. Der Rücklauf der Unterlagen von 407 Unternehmen stellte 20,35\% dar und bei Organisationen mit 200 und mehr Mitarbeitern erreichte er 
12,5 \%. Im Jahre 1998 wurde der Fragebogen an 407 Organisationen versandt, und der Rücklauf erreichte $42,75 \%$, d. h. die Unterlagen kamen von 174 Befragten zurück; bei Organisationen mit 200 und mehr Mitarbeitern waren es $37,6 \%$.

Durch die Untersuchungen wurde folgendes festgestellt:

die Mitgliedschaft in den Gewerkschaften nahm ständig ab,

soweit die Gewerkschaften im Unternehmen funktionieren, werden sie zum größten Teil als Partner der Tarifverhandlungen anerkannt,

es setzt sich eine starke Tendenz zur Dezentralisierung der Tarifverhandlung durch,

die Gewerkschaften des Unternehmens beteiligen sich gewöhnlich am Prozeß der Gestaltung der Lohnsysteme,

der Lohn besteht durchschnittlich zu 20-30 \% aus dem Leistungslohn,

die Kompetenzen des direkten Vorgesetzten nehmen hinsichtlich der Vergütung zu.

Über die Entlohnung ist zusammenfassend folgendes zu sagen:

Arbeitnehmer werden am häufigsten durch den Zeitlohn vergütet, der durch Prämien oder Leistungszulagen ergänzt wird. Bei der Leistungsmotivation werden die Prämien den Leistungszulagen vorgezogen. Hiermit möchte man vermeiden, daß der Vorgesetzte nach einer weitgehend subjektiven Mitarbeiterbeurteilung die Zulagen verteilt;

für Spezialisten und Techniker wird ungefähr bei der Hälfte der Befragten ein individuelles Gehalt vereinbart. Seine weitgehende Anwendung ist die Konsequenz der überhöhten Nachfrage nach diesen Professionsn sowie der verhältnismäßig beträchtlichen Limite in der Tarifvereinbarung. Bei der zweiten Hälfte der Unternehmen wird der Zeitlohn gewährt, der hauptsächlich durch Prämien ergänzt wird;

bei Managern überwiegt die außertarifliche Vergütung. Bei niedrigeren und mittleren Managern ist der Anteil des garantierten Gehalts verhältnißmäßig höher als bei Topmanagern. Die variablen Bestandteile des Gehalts werden vorwiegend durch Bonus, Erfolgsbeteiligung und Tantieme ergänzt.

Die liberalisierte Lohnpolitik in der Tschechischen Republik trug zur Erhöhung der Lohnkultur bei, denn sie schuf die Voraussetzungen dafür, daß die leistungsorientierte Lohngestaltung die Unternehmenspolitik wirksamer unterstützt. Die Konkurrenz zwischen Arbeitgebern im Kampf um die besseren Arbeitnehmer übte auf die Lohnsteigerung und die Erweiterung der Lohnunterschiede zwischen den Unternehmen Druck aus. 
Die Tarifverhandlungen stellten ein neues Element in der Industriebranche dar. Die Legislative zu deren Prozeßregelung wurde mit einer gewissen Verspätung erlassen. Die Sozialpartner bildeten sich mit unterschiedlicher Geschwindigkeit heraus und machten sich mit ihren Rollen vertraut. Die relativ hohe Organisiertheit in den Gewerkschaften führte am Anfang zum Abschluß solcher betrieblichen Tarifverträge, die für die Arbeitgeber mehr Verbindlichkeiten als Rechte enthielten. Das Management des Unternehmes hatte kein übersichtliches Statut und war bemüht, soziale Konflikte zu vermeiden. Deshalb wurden in den ersten Tarifverträgen Lohnforderungen und Vergünstigungen akzeptiert, die die ökonomischen Möglichkeiten der Unternehmen überstiegen. Die legislative Unklarheit bei der Übertragung der Gültigkeit der Tarifverträge, die zwischen dem Arbeitgeberverband und dem Gewerkschaftsverband vereinbart werden, auf Unternehmen der betreffenden Branche, die nicht Mitglied der Verbände sind, hatte zur Folge, daß die dezentralisierte Tarifverhandlung überwog. Die Entwicklung der Tarifverhandlung verlief trotz einigen ungünstigen Erscheinungen insgesamt positiv, was zur Erhaltung des sozialen Friedens beitrug.

Das Management der Unternehmen ist auf der Suche nach optimaler Lohngestaltung. Der sinkende Anteil des Tariflohns bei gewerblichen Arbeitern sowie Angestellten wird von vielen Firmen als ein wirksames Instrument der Leistungsmotivation angesehen. Sie sind der Meinung, daß nach den Jahren der niedrigen formellen und informellen Autorität der Vorgesetzten die neue Entscheidungskompetenz über einen relativ hohen variablen Lohnbestandteil die Stellung der Vorgesetzten stärken wird. Nach den Jahren der direktiven Führung stoßen die Arbeitgeber jedoch nur auf eine geringe Bereitschaft der Manager, für die Arbeitsergebnisse der untergeordneten Mirarbeiter Verantwortung zu tragen, besonders wenn es um die Bereitschaft der unmittelbaren Vorgesetzten in der Produktion geht. Probleme auf dem Arbeitsplatz umgehen sie manchmal durch die nivellierte Verteilung der variablen Lohnbestandteile. Ein solches Vorgehen läßt zu wünschen übrig. Leistungsstandards fehlen oder sind veraltet. Die systematische Beurteilung des Mitarbeiters wird meistens nach den Bedürfnissen des Bewerters durchgeführt. Dadurch kommt es zur unbegründeten Nivellierung der Löhne. Gegenwärtig sind neue Entwicklungstendenzen zu beobachten. Bei den Tarifverhandlungen erscheint die starke Tendenz, sich über den durchaus dominanten Anteil des Lohntarifs zu einigen. Das Management bemüht sich, die Effektivität der Leistungsanreize zu erhöhen, und die großen Unternehmen analysieren regelmäßig das inner- und überbetriebliche Lohngefälle.

\section{Literaturverzeichnis}

Flanagan, R.J. (1995): Changing Wage Structures in the Czech Republic, in: World Economic Outlook. A Survey of the Staff of the IMF, Washington, DC, IMF, p. 64. 
Klain, Z. (1995): Remuneration in the Czech Republic, in: Benefits \& Compensation International, Vol. 24, Iss. 8, pp. 10-15.

Mzdy zaměstnanců za rok 1996 a 1997 (1997, 1998) (Löhne der Mitarbeiter in den Jahren 1996 und 1997), Praha, ČSÚ. 